based on clinical observation and Dr. Tredgold has been careful to insist that ' our knowledge of antenatal pathology is still limited.'

In the chapter on moral deficiency the author deals with one of the four classes defined by the Mental Deficiency Act-the moral defective-a conception which has caused so much difficulty to doctors, students and administrators. This chapter clarifies the various possibilities in diagnosis that arise when the doctor is presented with a juvenile delinquent. The author does conclude that there is a rare class of case which shows moral defect due to 'a defect of mind which is permanent and incurable.' He points out that many so-called moral defectives have acquired a wrong label and that misconduct or delinquency has, in fact, arisen from environmental or psychological causes. Dr. Tredgold approves of the juvenile court, the guidance clinics or departments of child psychiatry and of the work of psychiatrists in prisons. It is his opinion that the extension of such facilities will eventually produce a diminution in crime "provided they are kept on sane and proper lines and are not allowed to develop into sentimental fads.' Every experienced psychiatrist will welcome these words.

The legal relations of mental deficiency are dealt with so that the enquirer can ,find readily the answers to his queries, be they medical or administrative. Mental deficiency and Education Acts are brought up to date and there is a clear section on educationally subnormal children which contains the famous follow up of 9,218 such children passing through the special schools of the City of Birmingham.

The chapter on the social implications of mental deficiency and what it may mean to civilized communities should be read and digested by everyone with an interest in the future of civilized countries. It contains a reasoned plea for the development of a social sentiment or conscience concerning marriage and reproduction by the diseased, the degenerate, the chronic criminal and the habitual pauper. Dr. Tredgold points out that it is to an informed medical profession that the State and the public must look for guidance and advice.

Tredgold's work should be possessed by every doctor. It is a necessity to the medical student and the graduate taking a higher qualification in psychiatry. Intelligent lay people with an interest in sociology will find it an extremely useful book of reference. It is a work closely packed with learning, the gathering of a long and busy life.

It would be unkind not to mention the publishers, Messrs. Ballière, Tindall and Cox, and to offer them an expression of sympathy on the close of a long and successful association with a great medical 'teacher and author. This edition is beautifully printed and bound. Although it contains more pages the book is actually less bulky than the reviewer's well-thumbed fifth edition.

G.W.B.J.

\section{ANATOMY OF THE AUTONOMIC NERVOUS SYSTEM}

By G. A. G. Mitchell, O.B.E., T.D., M.B., Ch.M., D.Sc. Pp. xvi +356 , with $\mathrm{r}_{3} 1$ illustrations, many in colour. Edinburgh: E. \& S. Livingstone Ltd. 1953. 55 s.

In this book, which, as the author states in his preface, is "primarily anatomical and concerned with man,' the word 'autonomic' is interpreted very broadly, and the field covered is correspondingly extensive. The first four chapters contain a historical survey and general accounts of the terminology, development and histology of autonomic nervous structures; they are followed by an account of certain regions of the central nervous system which may be concerned in autonomic function, including a description of the frontal lobes of the brain and their connexions, and a detailed summary of our present knowledge of the structural features of the hypothalamus. The remaining chapters, which form rather more than half of the book, are taken up with a description of the peripheral distribution of the sympathetic and parasympathetic nerves and plexuses. The illustrations are numerous and practically without exception of a very high standard. A full bibliography is given which will be of great value to those who wish to carry their studies further than is possible within the confines of a single volume.

Among the varied topics discussed two, at least, call for special notice. In the earlier chapters the description of the frontal lobes of the brain and of the hypothalamus is based on recent work, much of which has not yet found its way into textbooks. It is scattered in a large number of papers, many from the Oxford School of Anatomy, and in collecting this information together, Professor Mitchell has performed a valuable service to those who have not the time or opportunity to consult-the original publications. In the later chapters, the results of Professor Mitchell's own researches; chiefly on the peripheral autonomic nerves and plexuses, forms the basis of much that is described. To anatomists Professor Mitchell's careful and meticulous work in this difficult field is well known. The fact that so much has been checked, or in some cases recorded for the first time, as a result of the author's personal observations on human material, gives these chapters an authority which is not often found in similar descriptions. The work on which they are based is a substantial contribution to knowledge, and it is pleasant to find it so clearly described and illustrated in a single volume which will be easily accessible.

As was said, the term 'autonomic' is interpreted very broadly and one cannot escape the feeling that some of the less satisfactory features of the book are a result of this. Langley's original and precise definition, as an efferent system to visceral structures, consisting of pre- and post-ganglionic neurones connected by a synapse outside the central 
nervous system, is not adhered to. Instead the autonomic nervous system is defined as regulating processes not under voluntary or volitional control. This is to adopt a physiological, perhaps a psychological criterion, and would have justified a more adequate discussion of the functional aspects of the system, without which, much of the anatomy has little significance to the clinician. The author also seems to be on somewhat insecure ground in his extension of the term ' autonomic' to include a wide variety of afferent neurones. There is surely nothing ' irrational ' or 'whimsically inconsistent,' to quote his own words, in limiting the term autonomic to an efferent system precisely defined in structural terms. To do so is not to deny the existence of numerous afferent fibres from viscera, or of afferent components in such nerves as the vagus or the splanchnic branches of the sympathetic. It may be that afferent fibres from viscera are less widely recognized than they should be, but it is possible to emphasize their presence without extending the use of the term autonomic so that it loses any precise or definable meaning.

Another result of this very broad interpretation of the meaning of terms, is the inclusion of several topics which to many readers will seem irrelevant to the main purpose of the book. The descriptions given, for example, of olfactory and gustatory systems are admirable in themselves, and contain the results of much recent work not readily accessible in textbooks, but it is a little difficult to see why either of these special sensory systems should be classified as autonomic and parasympathetic. The argument given on page 147 in favour of this usage seems particularly weak and could be used to justify the inclusion of almost any afferent nerve in the autonomic system. There would seem to be even less justification for the inclusion of some of the other subjects, such as descriptions and figures of the sulci and gyri of the cerebral cortex, the cortical distribution of cerebral arteries and a number of others, most of which are described in greater detail in the standard textbooks of anatomy.

The criticism that a book contains more than one would expect to find, is not perhaps very serious. In the autonomic nervous system there are many features which are incompletely understood and much of a controversial nature, and there is room for disagreement over what should be included in it. While many will not agree with all the views expressed by Professor Mitchell, this book provides a comprehensive account of the anatomy of the innervation of viscera both efferent and afferent, considered from a broad but almost exclusively anatomical point of view. As such it is a valuable addition to medical literature; its controversial aspects will stimulate interest and, it may be hoped, further work, not only from anatomists but from physiologists and clinicians as well. It can be only from the co-operation of all three that the many problems of the autonomic nervous system will eventually be solved.

F.G.

\section{DISEASES OF THE CHEST}

Vols. I and II

Edited by Sir Geoffrey Marshall, K.C.V.O., C.B.E., M.D., F.R.C.P., and KENNETH M. A. Perry, M.A., M.D., F.R.C.P. Pp. xi +475 , with ${ }_{5} 8$ illustrations, and pp. vii +444 , with I 92 illustrations, respectively. London: Butterworth \& Co., Ltd. 1952. $\& 7$ 7s. the set.

Thirty-one experts have contributed to this textbook in two volumes which covers the whole field of chest diseases including tuberculosis. This is a formidable undertaking since books of equal length have been written on pulmonary tuberculosis alone.

It is claimed by the publishers that an even balance has been maintained between the sections concerning tuberculosis and other diseases; 208 pages have been devoted to the former and 657 to the latter. It is difficult to decide the relative importance of these two groups which may well change rapidly in the near future, but many chest specialists would probably suggest a more cven distribution. The tuberculosis section, although good, appears rather cramped and concerning treatment there is no mention of some important practical points, such as when to abandon an artificial pneumothorax especially in the presence of pleural thickening. The absence of this complication in pneumoperitoneum treatment is surely a great advantage. The period of maintenance of pneumoperitoneum recommended is two to three years - a rather short span for a less efficient form o collapse than artificial pneumothorax for which thẹ usual recommendation is five years.

The variability of the styles of the writers is striking, but is not distracting in the non-tuberculous sections where change of style usually coincides with change of disease. In the tuberculous section, however, these changes are rather disturbing to continuity of thought and may be confusing to the reader inexperienced in pulmonary tuberculosis.

In a book of this standard it is difficult to pick out sections for special praise and every critical reader would no doubt select a different group. The chapters dealing with bronchopulmonary anatomy, emphysema, applied physiology, haematogenous and pleural tuberculosis, pulmonary embolism and lung tumours must rank high on any list, and that dealing with asthma, a notoriously complex and 'partisan' disease, is a wise and balanced review.

The book is first class and fills a long-felt gap in medical literature. It is authoritative, full, practical and as up to date as possible. It is written almost entirely from personal experience and owes little or nothing to previous textbooks. As a whole, it is suitable as a reference book for chest specialists and it is therefore suggested that in future editions more space should be devoted to pulmonary tuberculosis, especially regarding treatment. Possibly this subject would be better treated by two authors - a physician and a surgeon.

The book is strongly recommended to membership candidates, general physicians and chest 